\title{
An Alternative Way to Relieve Neuropathic Pain After Total Knee Replacement by Genicular Nerve Radio-Frequency Ablation
}

\author{
Juzaily Fekry Leong*1, Rizal Abdul Rani ${ }^{1}$, Mohd Shahir bin Anuar ${ }^{2}$, Mohamed Ashraff Mohd Ariff ${ }^{1}$ and Nor Hamdan \\ Mohd Yahaya ${ }^{1}$ \\ ${ }^{1}$ Department of Orthopaedic and Traumatology, Universiti Kebangsaan Malaysia, Malaysia \\ ${ }^{2}$ Department of Anaesthesiology \& Intensive Care, Universiti Kebangsaan Malaysia, Malaysia
}

Received: 漹: September 03, 2018; Published: 䟧: October 09, 2018

*Corresponding author: Juzaily Fekry Leong, Department of Orthopaedic and Traumatology, Faculty of Medicine, Universiti Kebangsaan Malaysia, Hospital Canselor Tuanku Muhriz, Kuala Lumpur, Malaysia

\begin{abstract}
Total Knee Replacement (TKR) is a treatment of knee osteoarthritis to alleviate pain symptoms and improve physical functioning when other conservative treatments have failed. However, some patients still complaining of persistent post surgical pain (PPSP) after TKR. Although the cause of PPSP is not known, it seems that some of the patients with PPSP after TKR suffer from neuropathic pain. Recently genicular nerve radio-frequency ablation has emerged as an alternative intervention for patients at high risk who are unable to undergo surgical intervention. We have shown from our case, a 69-year old lady who had right total knee replacement presented with neuropathic knee pain has good symptom relieves after undergone radio-frequency ablation.
\end{abstract}

Keywords: Genicular Nerve; Chronic Pain; Radio-Frequency Ablation; Total Knee Replacement

Abbreviations: TKR: Total Knee Replacement; PPSP: Persistent Post Surgical Pain; RFA: Radio-Frequency Ablation; GN: Genicular Nerve; VAS: Visual Analogue Scale; SL: Superior Lateral; SM: Superior Medial; IM: Inferior Medial

\section{Introduction}

Pain in the knee joint can be from a variety of reasons. It can be either from the joint itself, it can be myofascial or it can be neuropathy, radicular pain. Total Knee replacement (TKR) is a treatment of osteoarthritis to relieve pain symptoms and improve physical function when conservative treatments have failed. However, not all patients are satisfied after TKR. Pain after TKR is stronger determinant of satisfaction than function [1]. Studies have shown that unfavorable pain outcome was seen in at least $8 \%$ and up to $26.5 \%$ of patients after TKR [2]. Apart from the post-surgical pain that is a result of a specific cause, some patients suffer from a persistent post-surgical pain (PPSP) with no specific origin. Although cause of PPSP is not known, it seem that some of the patients with PSSP after TKR suffer from the neuropathic pain. Recently genicular nerve radio-frequency ablation (RFA) has recently gained popularity as an intervention for chronic knee pain in patients who failed conservative or high risk to undergo Total Knee Replacement (TKR). By severing the nerve supply, the transmission of pain signals is disrupted, which will relieve pain and restore function. Prior giving RFA treatment, prognostic nerve blocks with local anesthetics at the site of pain generators have shown successful outcome.

\section{Case Report}

We present a case of a 69 years old lady who had right total knee replacement in December 2014. Post operatively, she complained of persistent right knee pain more in the anterior aspect of the knee. She needs to use [1] walking stick to help with her ambulation. Extensive conservative treatments in the form of medication, physical therapy and injections including steroid were unsuccessful. Blood investigation, radiology and knee fluid sample taken to investigate for infection were negative. Given the patient's symptoms and the failure of conservative intervention, diagnostic nerve block was performed before proceeding with RFA. Genicular nerve block was performed by our anaesthetist. Under sterile conditions, three spinal needles $22 \mathrm{G}$ were placed under fluoroscopy (Figure 1). To block the genicular nerve (GN) brunches, a cocktail of $0.2 \%$ ropivacaine and $40 \mathrm{mg}$ of triamcinolone were injected at right superior medial, superior lateral and inferior medial brunch of GN. Her visual analogue scale (VAS) improved from 8 to 3 after 12 hours of the procedure. The pain relief lasted for 6 weeks and based on this result, patient then proceeded with RFA. Post RFA, patient symptom improves more than $50 \%$ and was able to walk without walking aid. 


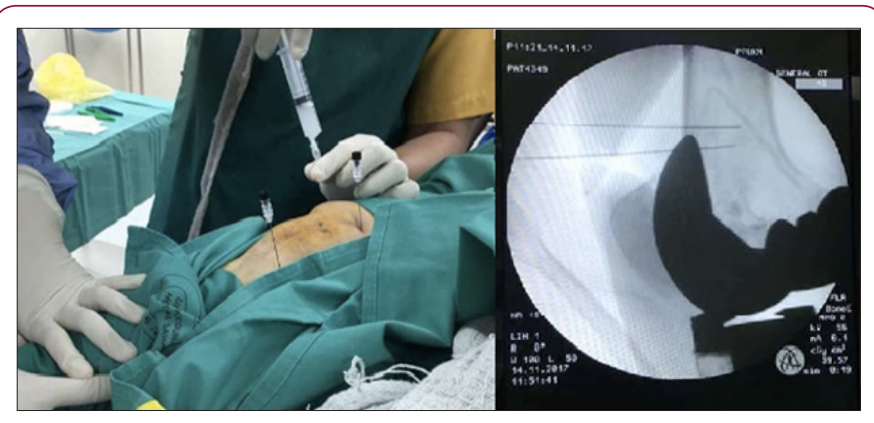

Figure 1: Procedure needle placement under fluoroscopy imaging.

\section{Discussion}

The knee joint is innervated by the articular branches of various nerves including the femoral, common peroneal, saphenous, tibial and obturator nerves. This articular branches around the knee joint are known as genicular nerves [3]. Anatomical study by Callaghan et al. [4], the Superior lateral (SL) genicular nerve originates from the common peroneal division of the sciatic nerve $8-10 \mathrm{~cm}$ superior to the joint line. It travels toward the superolateral aspect of the knee capsule deep to the biceps femoris muscle and the iliotibial band. The tibial nerve gives rise to 3 articular branches in the popliteal fossa, 2 of which are located on the medial aspect of the knee joint and are targets of RFA: the Superior medial (SM) and Inferior medial (IM) genicular nerves. SL, SM and IM genicular nerves innervate the articular capsule of the knee joint and these nerves pass close to femur and tibia epicondyles and therefore are relatively precise in anatomic aspect for target point in radiofrequency [5]. Genicular nerves run in close proximity to genicular arteries that play a crucial role in supplying the distal femur, knee joint, meniscus and patella.

By using Ultrasound, neurovascular bundles can be identified and this allowed better visualization and presumably more accurate of nerve identification. Some reported that by using ultrasound, it reduces the technical difficulty in locating the nerves [6]. RFA has been used to treat a variety of pain conditions. It was originally used to treat trigeminal neuralgia and was expanded to address spinal pain [7]. More recently, investigators used this technique for the management of knee osteoarthritis pain for patients at high risk who could not undergo TKR. In 2011, Ikeuchi et al. [8] conducted a controlled trial to examine the efficacy of RFA application to sensory nerves. Twelve weeks after the intervention, the patients treated with RF had greater improvements in pain compared with the control group [8]. Similarly, Choi et al. [5] investigated the use of RFA treatment to alleviate chronic knee osteoarthritis pain. The randomized controlled trial demonstrated greater improvements in pain, function, and satisfaction for patients treated with RF than for controls [5]. Although the follow-up was limited to 12 weeks, analysis of these findings suggests that RFA neurotomy of the GNs has the potential to significantly improve pain, function, and satisfaction [5].

In conclusion, we have shown in our case, RFA can be applied not only to patients who failed conservative treatment or high risk of surgical procedures but also to those who already had TKR. So RFA can be one of our treatment choice in managing chronic knee pain patients post total knee replacement.

\section{References}

1. Drosos GI, Triantafilidou T, Ververidis A (2015) Persistent post-surgical pain and neuropathic pain after total knee replacement. World J Orthop 6(7): 528-536.

2. Beswick PN, Van Der meulen JH, Lewsey J (2007) The role of pain and function in determining patient satisfaction after total knee replacement. Data from the National Joint Registry for England and Wales. J Bone Joint Surg Br 89(7): 893-900.

3. Carlo CD, Buvanendran A, Petersohn J (2015) Innervation of the Anterior Capsule of the Human Knee. Regional Anethesia and Pain Medicine 40(4): 363-368.

4. Callaghan JJ, Rosenberg AG, Rubash HE (2003) Surgical Anatomy of the Knee. In: The Adult Knee. Wasielewski RC (Eds.) Lip- pincott Williams \& Wilkins, Philadelphia p. 77-78.

5. Choi WJ, Hwang SJ, Song JG, Leem JG, Kang YU, et al. (2011) Radiofrequency treatment relieves chronic knee osteoarthritis pain: A double-blind randomized controlled trial. Pain 152: 481-487.

6. Bhatia A, Peng P, Cohen SP (2016) Radiofrequency procedures to relieve chronic knee pain. An evidence-based narrative review. Regional Anesthesia and Pain Medicine 41(4): 501-510.

7. Bogduk N (2006) Pulsed radiofrequency. Pain med 7: 396-407.

8. Ikeuchi M, Ushida T, Izumi M, Tani T (2011) Percutaneous radiofrequency treatment for refactory anteromedial pain of osteoarthritis knees. Pain Med 12(4): 546-551.
ISSN: 2574-1241

DOI: $10.26717 /$ BJSTR.2018.09.001845

Juzaily Fekry Leong. Biomed J Sci \& Tech Res

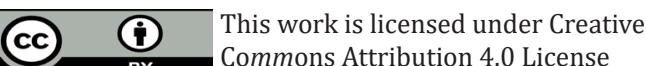

Submission Link: https://biomedres.us/submit-manuscript.php

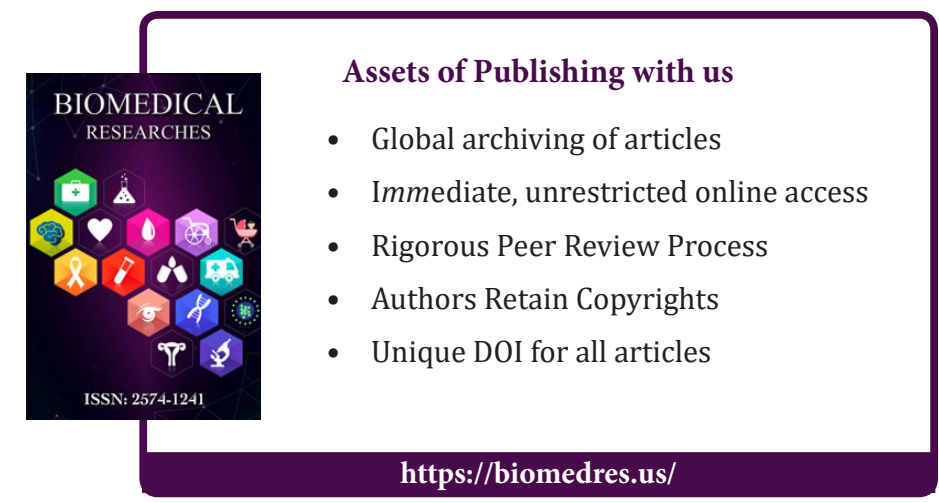

